\title{
Substituent Effects on the Self-Assembly/Coassembly and Hydrogelation of Phenylalanine Derivatives
}

\author{
Wathsala Liyanage and Bradley L. Nilsson* \\ Department of Chemistry, University of Rochester, Rochester, NY, 14627-0216, USA. \\ E-mail: nilsson@chem.rochester.edu \\ Supporting Information, Appendix 1 \\ CRYSTAL STRUCTURE REPORT \\ $\mathrm{C}_{24.7} \mathrm{H}_{20} \mathrm{~N}_{2} \mathrm{O}_{4.6}$ \\ or \\ $[\text { Fmoc-4CN-Phe-OH }]_{0.7} \cdot[\text { Fmoc-4NO} 2-P h e-O H]_{0.3}$ \\ Report prepared for: \\ W. Liyanage, Prof. B. Nilsson
}

March 12, 2012

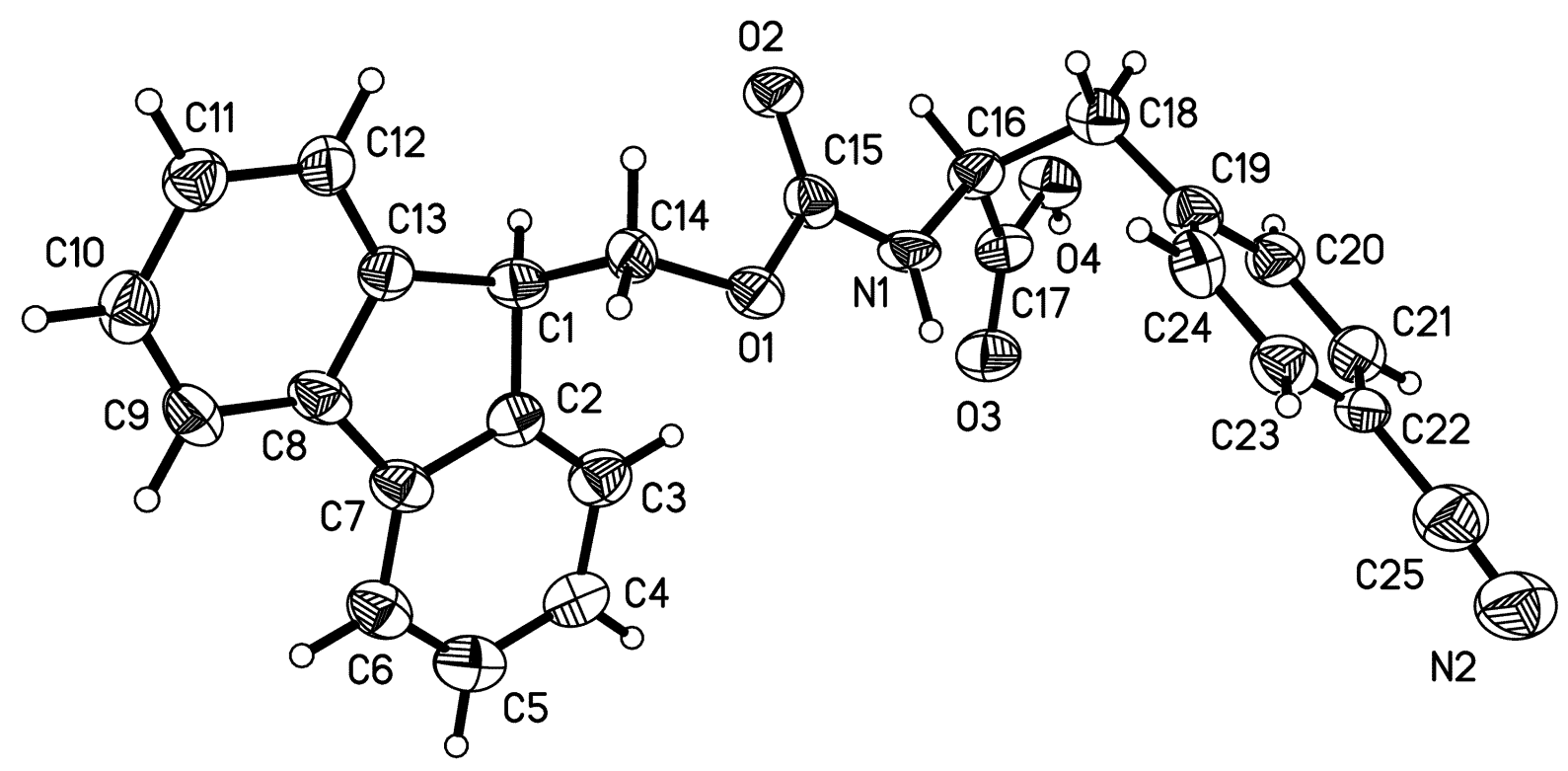

William W. Brennessel

X-ray Crystallographic Facility

Department of Chemistry, University of Rochester

120 Trustee Road

Rochester, NY 14627 


\section{Data collection}

A crystal $\left(0.28 \times 0.08 \times 0.04 \mathrm{~mm}^{3}\right)$ was placed onto the tip of a $0.1 \mathrm{~mm}$ diameter glass capillary tube or fiber and mounted on a Bruker SMART APEX II CCD Platform diffractometer for a data collection at 100.0(5) K. ${ }^{1} \mathrm{~A}$ preliminary set of cell constants and an orientation matrix were calculated from reflections harvested from three orthogonal wedges of reciprocal space. The full data collection was carried out using MoK $\alpha$ radiation (graphite monochromator) with a frame time of 120 seconds and a detector distance of $4.01 \mathrm{~cm}$. A randomly oriented region of reciprocal space was surveyed: four major sections of frames were collected with $0.50^{\circ}$ steps in $\omega$ at four different $\phi$ settings and a detector position of $-38^{\circ}$ in $2 \theta$. The intensity data were corrected for absorption. ${ }^{2}$ Final cell constants were calculated from the xyz centroids of 1306 strong reflections from the actual data collection after integration. $^{3}$ See Table 1 for additional crystal and refinement information.

\section{Structure solution and refinement}

The structure was solved using SIR $97^{4}$ and refined using SHELXL-97. ${ }^{5}$ The space group $P 2{ }_{1} 2_{1} 2_{1}$ was determined based on systematic absences. A direct-methods solution was calculated which provided most non-hydrogen atoms from the E-map. Full-matrix least squares / difference Fourier cycles were performed which located the remaining non-hydrogen atoms. All non-hydrogen atoms were refined with anisotropic displacement parameters. All hydrogen atoms were placed in ideal positions and refined as riding atoms with relative isotropic displacement parameters. Due to the lack of significant anomalous dispersion effects, the correct enantiomer was assigned (provided by the chemist) and all Friedel opposites were merged in the final refinement. The final full matrix least squares refinement converged to $R 1=0.0601\left(F^{2}, I>2 \sigma(I)\right)$ and $w R 2=0.1428\left(F^{2}\right.$, all data). Due to the poor quality of the intensity data, it is suggested that the crystals be regrown from different media.

\section{Structure description}

The structure is one of the ones suggested, with all atoms in general positions. The structure is a cocrystallization of two independent species, one with a cyano group and one with a nitro group at the para position of the phenyl ring (70:30, respectively). The structure is a one-dimensional polymer via hydrogen bonding (see diagrams and Table 7).

Unless noted otherwise all structural diagrams containing thermal displacement ellipsoids are drawn at the $50 \%$ probability level.

Data collection, structure solution, and structure refinement were conducted at the X-ray Crystallographic Facility, B51 Hutchison Hall, Department of Chemistry, University of Rochester. All publications arising from this report MUST either 1) include William W. Brennessel as a coauthor or 2) acknowledge William W. Brennessel and the Xray Crystallographic Facility of the Department of Chemistry at the University of Rochester. 
1 APEX2, version 2011.4-1; Bruker AXS: Madison, WI, 2011.

2 Sheldrick, G. M. SADABS, version 2008/1; University of Göttingen: Göttingen, Germany, 2008.

3 SAINT, version 7.68A; Bruker AXS: Madison, WI, 2009.

4 Altomare, A.; Burla, M. C.; Camalli, M.; Cascarano, G. L.; Giacovazzo, C.; Guagliardi, A.; Moliterni, A. G. G.;

Polidori, G.; Spagna, R. SIR97: A new program for solving and refining crystal structures; Istituto di Cristallografia, CNR: Bari, Italy, 1999.

5 Sheldrick, G. M. Acta. Cryst. 2008, A64, 112-122.

Some equations of interest:

$$
\begin{gathered}
R_{\mathrm{int}}=\Sigma\left|F_{\mathrm{o}}{ }^{2}-<F_{\mathrm{o}}{ }^{2}>\right| / \Sigma\left|F_{\mathrm{o}}{ }^{2}\right| \\
R 1=\Sigma|| F_{\mathrm{o}}|-| F_{\mathrm{c}} \| / \Sigma\left|F_{\mathrm{o}}\right| \\
w R 2=\left[\Sigma\left[w\left(F_{\mathrm{o}}{ }^{2}-F_{\mathrm{c}}{ }^{2}\right)^{2}\right] / \Sigma\left[w\left(F_{\mathrm{o}}{ }^{2}\right)^{2}\right]\right]^{1 / 2} \\
\text { where } w=1 /\left[\sigma^{2}\left(F_{\mathrm{o}}{ }^{2}\right)+(a P)^{2}+b P\right] \text { and } \\
P=1 / 3 \max \left(0, F_{\mathrm{o}}{ }^{2}\right)+2 / 3 F_{\mathrm{c}}{ }^{2} \\
\mathrm{GOF}=S=\left[\Sigma\left[w\left(F_{\mathrm{o}}{ }^{2}-F_{\mathrm{c}}{ }^{2}\right)^{2}\right] /(m-n)\right]^{1 / 2}
\end{gathered}
$$

where $m=$ number of reflections and $n=$ number of parameters

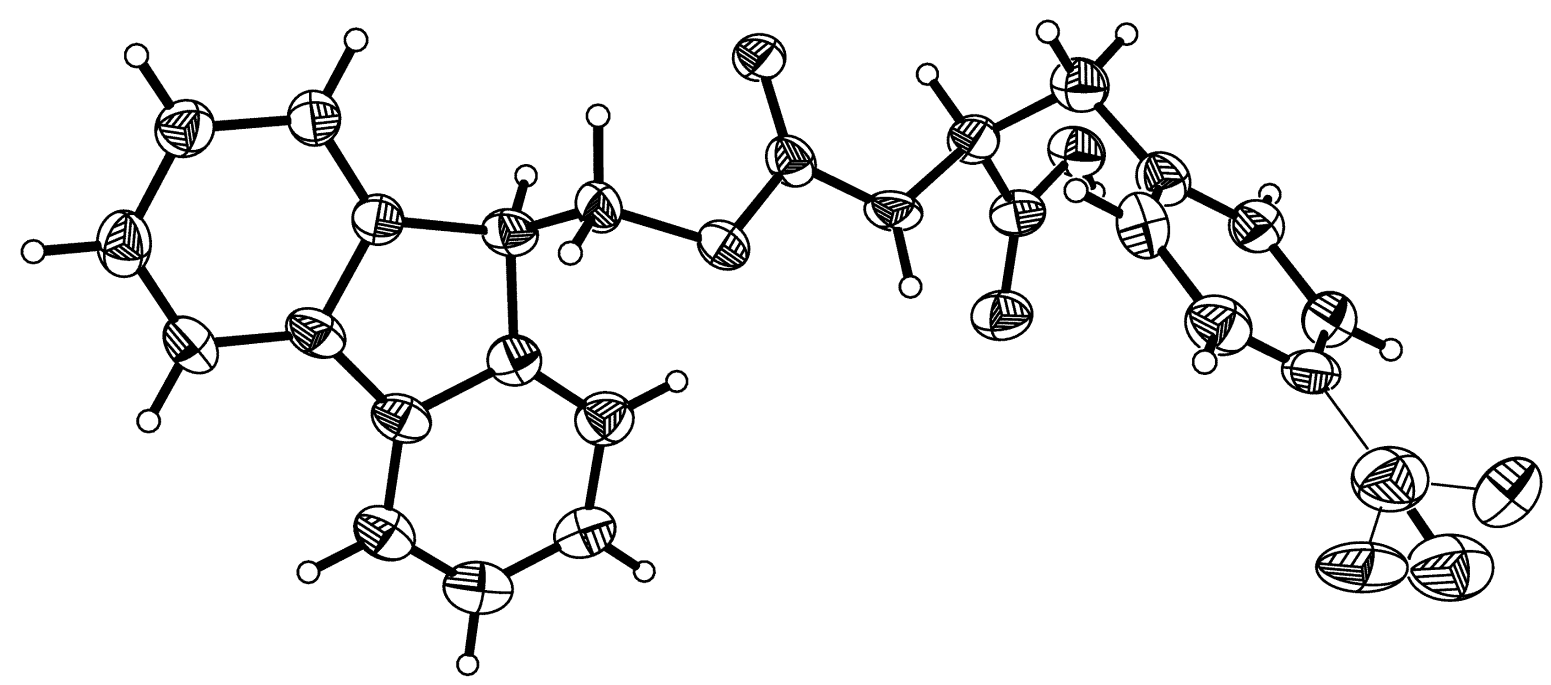



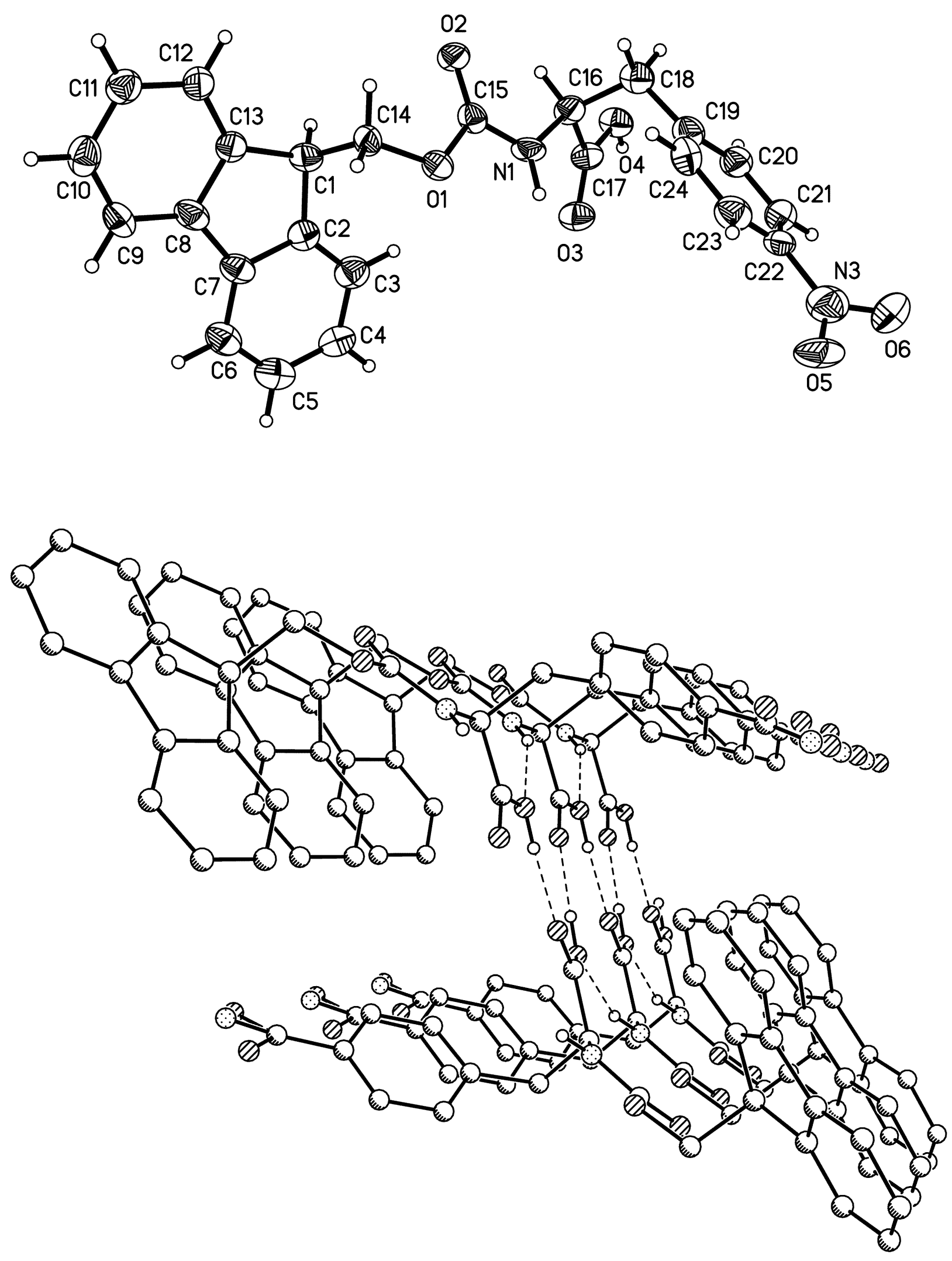


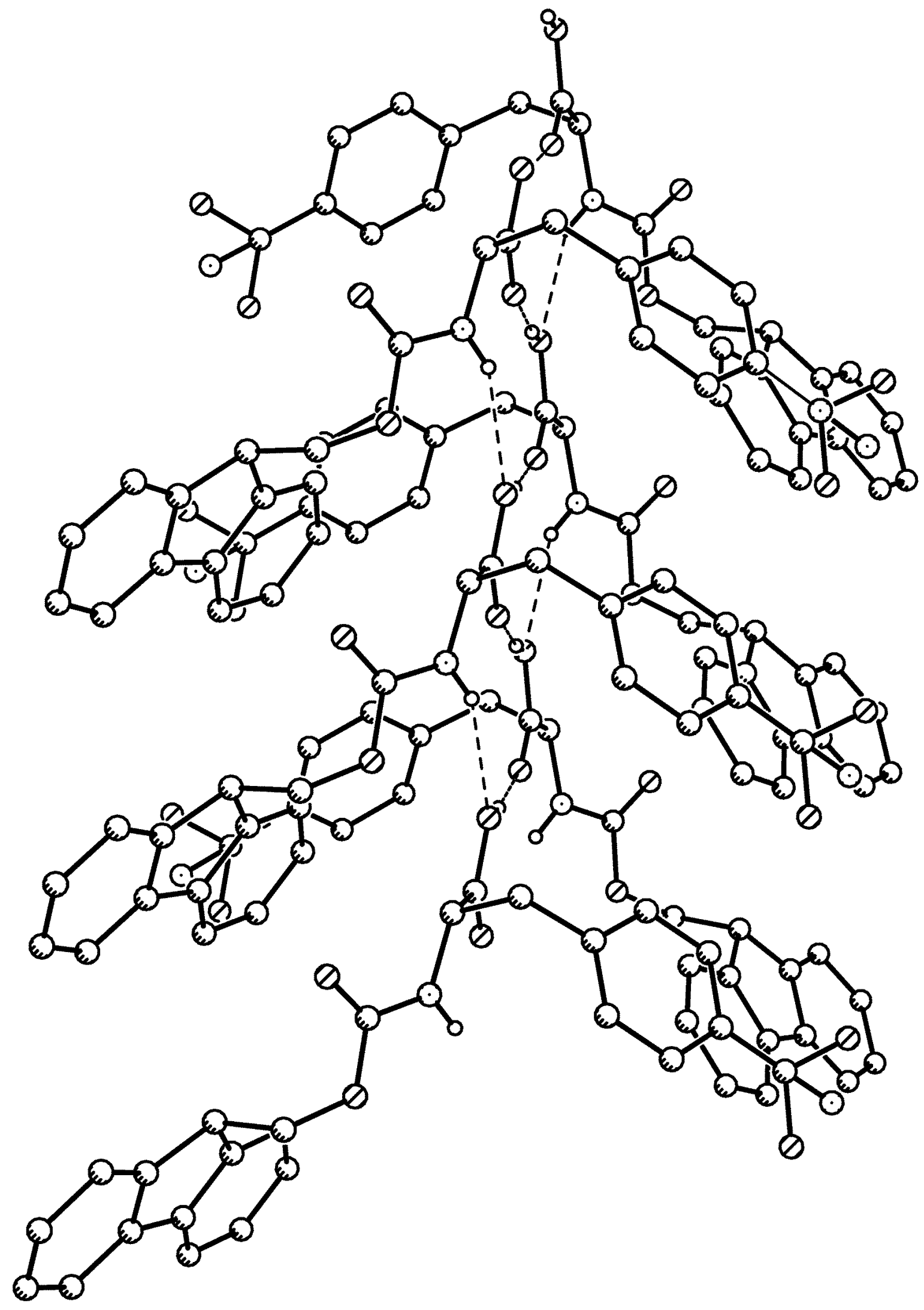


Table 1. Crystal data and structure refinement for nilw106.

\begin{tabular}{|c|c|c|}
\hline Identification code & \multicolumn{2}{|l|}{ nilw106 } \\
\hline Empirical formula & \multicolumn{2}{|c|}{$\mathrm{C} 24.7 \mathrm{H} 20 \mathrm{~N} 2 \mathrm{O} 4.6$} \\
\hline Formula weight & \multicolumn{2}{|l|}{418.42} \\
\hline Temperature & \multicolumn{2}{|l|}{$100.0(5) \mathrm{K}$} \\
\hline Wavelength & \multicolumn{2}{|l|}{$0.71073 \AA$} \\
\hline Crystal system & \multicolumn{2}{|l|}{ Orthorhombic } \\
\hline Space group & \multicolumn{2}{|l|}{$P 2_{1} 2_{1} 2_{1}$} \\
\hline \multirow[t]{3}{*}{ Unit cell dimensions } & $a=5.747(3) \AA$ & $\alpha=90^{\circ}$ \\
\hline & $b=15.850(7) \AA$ & $\beta=90^{\circ}$ \\
\hline & $c=22.201(10) \AA$ & $\gamma=90^{\circ}$ \\
\hline Volume & \multicolumn{2}{|l|}{$2022.2(16) \AA^{3}$} \\
\hline$Z$ & \multicolumn{2}{|l|}{4} \\
\hline Density (calculated) & \multicolumn{2}{|l|}{$1.374 \mathrm{Mg} / \mathrm{m}^{3}$} \\
\hline Absorption coefficient & \multicolumn{2}{|l|}{$0.096 \mathrm{~mm}^{-1}$} \\
\hline$F(000)$ & \multicolumn{2}{|l|}{876} \\
\hline Crystal color, morphology & \multicolumn{2}{|l|}{ colorless, needle } \\
\hline Crystal size & \multicolumn{2}{|c|}{$0.28 \times 0.08 \times 0.04 \mathrm{~mm}^{3}$} \\
\hline Theta range for data collection & \multicolumn{2}{|l|}{1.83 to $25.02^{\circ}$} \\
\hline Index ranges & \multicolumn{2}{|c|}{$-6 \leq h \leq 6,-18 \leq k \leq 18,-26 \leq l \leq 26$} \\
\hline Reflections collected & \multicolumn{2}{|l|}{20557} \\
\hline Independent reflections & \multicolumn{2}{|c|}{$2087[R(\mathrm{int})=0.2403]$} \\
\hline Observed reflections & \multicolumn{2}{|l|}{1233} \\
\hline Completeness to theta $=25.02^{\circ}$ & \multicolumn{2}{|l|}{$100.0 \%$} \\
\hline Absorption correction & \multicolumn{2}{|l|}{ Multi-scan } \\
\hline Max. and min. transmission & \multicolumn{2}{|l|}{0.9962 and 0.9737} \\
\hline Refinement method & \multicolumn{2}{|c|}{ Full-matrix least-squares on $F^{2}$} \\
\hline Data / restraints / parameters & \multicolumn{2}{|l|}{$2087 / 12 / 300$} \\
\hline Goodness-of-fit on $F^{2}$ & \multicolumn{2}{|l|}{1.026} \\
\hline Final $R$ indices $[I>2 \operatorname{sigma}(I)]$ & \multicolumn{2}{|c|}{$R 1=0.0601, w R 2=0.1163$} \\
\hline$R$ indices (all data) & \multicolumn{2}{|c|}{$R 1=0.1257, w R 2=0.1428$} \\
\hline Extinction coefficient & \multicolumn{2}{|l|}{$0.022(3)$} \\
\hline Largest diff. peak and hole & \multicolumn{2}{|c|}{0.273 and -0.241 e. $\AA^{-3}$} \\
\hline
\end{tabular}


Table 2. Atomic coordinates $\left(\mathrm{x} 10^{4}\right)$ and equivalent isotropic displacement parameters $\left(\AA^{2} \times 10^{3}\right)$ for nilw106. $U_{\text {eq }}$ is defined as one third of the trace of the orthogonalized $U_{i j}$ tensor.

\begin{tabular}{|c|c|c|c|c|}
\hline & $\mathrm{x}$ & $\mathrm{y}$ & $\mathrm{z}$ & $\mathrm{U}_{\mathrm{eq}}$ \\
\hline $\mathrm{O} 1$ & $2604(6)$ & $206(2)$ & $1407(2)$ & $36(1)$ \\
\hline $\mathrm{O} 2$ & $-1062(7)$ & $-258(2)$ & $1580(2)$ & $36(1)$ \\
\hline $\mathrm{O} 3$ & $-1702(8)$ & 2124(3) & $355(2)$ & $44(1)$ \\
\hline $\mathrm{O} 4$ & $-5488(7)$ & 2005(3) & $611(2)$ & $40(1)$ \\
\hline N1 & $-351(8)$ & 1077(3) & $1248(2)$ & $35(1)$ \\
\hline $\mathrm{C} 1$ & $3632(11)$ & $-1151(4)$ & $940(3)$ & $33(2)$ \\
\hline $\mathrm{C} 2$ & $4953(10)$ & $-720(4)$ & $430(3)$ & $32(2)$ \\
\hline $\mathrm{C} 3$ & $4443(12)$ & $4(4)$ & $112(3)$ & $42(2)$ \\
\hline $\mathrm{C} 4$ & $6003(12)$ & 274(4) & $-328(3)$ & $42(2)$ \\
\hline $\mathrm{C} 5$ & $8002(12)$ & $-182(4)$ & $-452(3)$ & $46(2)$ \\
\hline C6 & $8507(12)$ & $-914(4)$ & $-149(3)$ & $41(2)$ \\
\hline $\mathrm{C} 7$ & $7000(10)$ & $-1184(4)$ & 293(3) & $35(2)$ \\
\hline $\mathrm{C} 8$ & $7068(11)$ & $-1929(4)$ & $683(3)$ & $37(2)$ \\
\hline C9 & $8664(11)$ & $-2572(4)$ & $727(3)$ & $38(2)$ \\
\hline $\mathrm{C} 10$ & $8303(12)$ & $-3215(4)$ & 1134(3) & $42(2)$ \\
\hline $\mathrm{C} 11$ & $6340(11)$ & $-3228(4)$ & $1495(3)$ & $40(2)$ \\
\hline $\mathrm{C} 12$ & $4706(11)$ & $-2581(3)$ & 1453(3) & $37(2)$ \\
\hline $\mathrm{C} 13$ & $5054(10)$ & $-1933(4)$ & 1051(3) & $32(2)$ \\
\hline $\mathrm{C} 14$ & $3493(11)$ & $-632(3)$ & 1521(3) & $33(2)$ \\
\hline $\mathrm{C} 15$ & 252(10) & $296(4)$ & 1423(3) & $32(2)$ \\
\hline C16 & $-2813(10)$ & 1307(4) & $1227(3)$ & $37(2)$ \\
\hline $\mathrm{C} 17$ & $-3239(12)$ & $1848(4)$ & $680(3)$ & $37(2)$ \\
\hline $\mathrm{C} 18$ & $-3579(11)$ & 1772(4) & $1810(3)$ & $39(2)$ \\
\hline C19 & $-2335(11)$ & 2591(4) & 1907(3) & $37(2)$ \\
\hline $\mathrm{C} 20$ & $-3127(12)$ & $3337(4)$ & $1663(3)$ & $41(2)$ \\
\hline $\mathrm{C} 21$ & $-1889(12)$ & 4071(4) & 1723(3) & $42(2)$ \\
\hline $\mathrm{C} 22$ & $206(12)$ & $4062(4)$ & $2038(3)$ & $40(2)$ \\
\hline $\mathrm{C} 23$ & $1003(12)$ & $3338(4)$ & $2298(3)$ & $43(2)$ \\
\hline $\mathrm{C} 24$ & $-267(12)$ & 2597(4) & $2234(3)$ & $42(2)$ \\
\hline $\mathrm{C} 25$ & $1524(16)$ & $4831(6)$ & 2081(3) & $59(2)$ \\
\hline N2 & $2510(20)$ & $5431(8)$ & $2066(4)$ & $67(3)$ \\
\hline
\end{tabular}




$\begin{array}{lrlll}\text { N3 } & 1524(16) & 4831(6) & 2081(3) & 59(2) \\ \text { O5 } & 3750(30) & 4878(14) & 2198(8) & 55(5) \\ \text { O6 } & 530(30) & 5569(11) & 2088(7) & 47(5)\end{array}$


Table 3. Bond lengths $[\AA]$ and angles $\left[{ }^{\circ}\right]$ for nilwlo6.

\begin{tabular}{|c|c|c|c|}
\hline $\mathrm{O}(1)-\mathrm{C}(15)$ & $1.360(7)$ & $\mathrm{C}(14)-\mathrm{H}(14 \mathrm{~A})$ & 0.9900 \\
\hline $\mathrm{O}(1)-\mathrm{C}(14)$ & $1.446(7)$ & $\mathrm{C}(14)-\mathrm{H}(14 \mathrm{~B})$ & 0.9900 \\
\hline $\mathrm{O}(2)-\mathrm{C}(15)$ & $1.210(7)$ & $\mathrm{C}(16)-\mathrm{C}(17)$ & $1.507(9)$ \\
\hline $\mathrm{O}(3)-\mathrm{C}(17)$ & $1.221(7)$ & $C(16)-C(18)$ & $1.553(8)$ \\
\hline $\mathrm{O}(4)-\mathrm{C}(17)$ & $1.326(7)$ & $\mathrm{C}(16)-\mathrm{H}(16)$ & 1.0000 \\
\hline $\mathrm{O}(4)-\mathrm{H}(4 \mathrm{O})$ & 0.8400 & $C(18)-C(19)$ & $1.498(8)$ \\
\hline $\mathrm{N}(1)-\mathrm{C}(15)$ & $1.343(7)$ & $\mathrm{C}(18)-\mathrm{H}(18 \mathrm{~A})$ & 0.9900 \\
\hline $\mathrm{N}(1)-\mathrm{C}(16)$ & $1.462(7)$ & $\mathrm{C}(18)-\mathrm{H}(18 \mathrm{~B})$ & 0.9900 \\
\hline $\mathrm{N}(1)-\mathrm{H}(1 \mathrm{~N})$ & 0.8800 & $C(19)-C(20)$ & $1.377(8)$ \\
\hline$C(1)-C(13)$ & $1.505(8)$ & $C(19)-C(24)$ & $1.393(8)$ \\
\hline $\mathrm{C}(1)-\mathrm{C}(2)$ & $1.525(8)$ & $\mathrm{C}(20)-\mathrm{C}(21)$ & $1.370(8)$ \\
\hline$C(1)-C(14)$ & $1.531(8)$ & $\mathrm{C}(20)-\mathrm{H}(20)$ & 0.9500 \\
\hline $\mathrm{C}(1)-\mathrm{H}(1)$ & 1.0000 & $C(21)-C(22)$ & $1.393(9)$ \\
\hline $\mathrm{C}(2)-\mathrm{C}(3)$ & $1.379(8)$ & $\mathrm{C}(21)-\mathrm{H}(21)$ & 0.9500 \\
\hline$C(2)-C(7)$ & $1.420(8)$ & $C(22)-C(23)$ & $1.363(9)$ \\
\hline $\mathrm{C}(3)-\mathrm{C}(4)$ & $1.394(9)$ & $C(22)-C(25)$ & $1.438(10)$ \\
\hline $\mathrm{C}(3)-\mathrm{H}(3)$ & 0.9500 & $C(23)-C(24)$ & $1.390(9)$ \\
\hline$C(4)-C(5)$ & $1.385(9)$ & $\mathrm{C}(23)-\mathrm{H}(23)$ & 0.9500 \\
\hline $\mathrm{C}(4)-\mathrm{H}(4)$ & 0.9500 & $\mathrm{C}(24)-\mathrm{H}(24)$ & 0.9500 \\
\hline$C(5)-C(6)$ & $1.372(9)$ & $\mathrm{C}(25)-\mathrm{N}(2)$ & $1.108(12)$ \\
\hline $\mathrm{C}(5)-\mathrm{H}(5)$ & 0.9500 & $\mathrm{C}(15)-\mathrm{O}(1)-\mathrm{C}(14)$ & $116.3(5)$ \\
\hline$C(6)-C(7)$ & $1.378(8)$ & $\mathrm{C}(17)-\mathrm{O}(4)-\mathrm{H}(4 \mathrm{O})$ & 109.5 \\
\hline $\mathrm{C}(6)-\mathrm{H}(6)$ & 0.9500 & $\mathrm{C}(15)-\mathrm{N}(1)-\mathrm{C}(16)$ & $119.2(5)$ \\
\hline$C(7)-C(8)$ & $1.464(8)$ & $\mathrm{C}(15)-\mathrm{N}(1)-\mathrm{H}(1 \mathrm{~N})$ & 120.4 \\
\hline $\mathrm{C}(8)-\mathrm{C}(9)$ & $1.374(8)$ & $\mathrm{C}(16)-\mathrm{N}(1)-\mathrm{H}(1 \mathrm{~N})$ & 120.4 \\
\hline $\mathrm{C}(8)-\mathrm{C}(13)$ & $1.418(8)$ & $C(13)-C(1)-C(2)$ & $102.7(5)$ \\
\hline$C(9)-C(10)$ & $1.379(8)$ & $C(13)-C(1)-C(14)$ & $109.4(5)$ \\
\hline $\mathrm{C}(9)-\mathrm{H}(9)$ & 0.9500 & $C(2)-C(1)-C(14)$ & $114.2(5)$ \\
\hline$C(10)-C(11)$ & $1.384(9)$ & $\mathrm{C}(13)-\mathrm{C}(1)-\mathrm{H}(1)$ & 110.1 \\
\hline $\mathrm{C}(10)-\mathrm{H}(10)$ & 0.9500 & $\mathrm{C}(2)-\mathrm{C}(1)-\mathrm{H}(1)$ & 110.1 \\
\hline $\mathrm{C}(11)-\mathrm{C}(12)$ & $1.393(8)$ & $\mathrm{C}(14)-\mathrm{C}(1)-\mathrm{H}(1)$ & 110.1 \\
\hline $\mathrm{C}(11)-\mathrm{H}(11)$ & 0.9500 & $C(3)-C(2)-C(7)$ & $119.8(6)$ \\
\hline$C(12)-C(13)$ & $1.374(8)$ & $C(3)-C(2)-C(1)$ & $130.3(6)$ \\
\hline $\mathrm{C}(12)-\mathrm{H}(12)$ & 0.9500 & $\mathrm{C}(7)-\mathrm{C}(2)-\mathrm{C}(1)$ & $109.8(5)$ \\
\hline
\end{tabular}




\begin{tabular}{|c|c|c|c|}
\hline$C(2)-C(3)-C(4)$ & $118.6(6)$ & $\mathrm{O}(1)-\mathrm{C}(14)-\mathrm{H}(14 \mathrm{~B})$ & 109.4 \\
\hline $\mathrm{C}(2)-\mathrm{C}(3)-\mathrm{H}(3)$ & 120.7 & $\mathrm{C}(1)-\mathrm{C}(14)-\mathrm{H}(14 \mathrm{~B})$ & 109.4 \\
\hline $\mathrm{C}(4)-\mathrm{C}(3)-\mathrm{H}(3)$ & 120.7 & $\mathrm{H}(14 \mathrm{~A})-\mathrm{C}(14)-\mathrm{H}(14 \mathrm{~B})$ & 108.0 \\
\hline $\mathrm{C}(5)-\mathrm{C}(4)-\mathrm{C}(3)$ & $120.8(6)$ & $\mathrm{O}(2)-\mathrm{C}(15)-\mathrm{N}(1)$ & $126.3(5)$ \\
\hline $\mathrm{C}(5)-\mathrm{C}(4)-\mathrm{H}(4)$ & 119.6 & $\mathrm{O}(2)-\mathrm{C}(15)-\mathrm{O}(1)$ & $123.5(5)$ \\
\hline $\mathrm{C}(3)-\mathrm{C}(4)-\mathrm{H}(4)$ & 119.6 & $\mathrm{~N}(1)-\mathrm{C}(15)-\mathrm{O}(1)$ & $110.2(5)$ \\
\hline$C(6)-C(5)-C(4)$ & $121.3(6)$ & $\mathrm{N}(1)-\mathrm{C}(16)-\mathrm{C}(17)$ & $108.9(5)$ \\
\hline $\mathrm{C}(6)-\mathrm{C}(5)-\mathrm{H}(5)$ & 119.3 & $\mathrm{~N}(1)-\mathrm{C}(16)-\mathrm{C}(18)$ & $111.5(5)$ \\
\hline $\mathrm{C}(4)-\mathrm{C}(5)-\mathrm{H}(5)$ & 119.3 & $C(17)-C(16)-C(18)$ & $110.9(5)$ \\
\hline$C(5)-C(6)-C(7)$ & $118.6(6)$ & $\mathrm{N}(1)-\mathrm{C}(16)-\mathrm{H}(16)$ & 108.5 \\
\hline $\mathrm{C}(5)-\mathrm{C}(6)-\mathrm{H}(6)$ & 120.7 & $\mathrm{C}(17)-\mathrm{C}(16)-\mathrm{H}(16)$ & 108.5 \\
\hline $\mathrm{C}(7)-\mathrm{C}(6)-\mathrm{H}(6)$ & 120.7 & $\mathrm{C}(18)-\mathrm{C}(16)-\mathrm{H}(16)$ & 108.5 \\
\hline$C(6)-C(7)-C(2)$ & $120.9(6)$ & $\mathrm{O}(3)-\mathrm{C}(17)-\mathrm{O}(4)$ & $124.7(6)$ \\
\hline $\mathrm{C}(6)-\mathrm{C}(7)-\mathrm{C}(8)$ & $130.9(6)$ & $\mathrm{O}(3)-\mathrm{C}(17)-\mathrm{C}(16)$ & $124.2(6)$ \\
\hline $\mathrm{C}(2)-\mathrm{C}(7)-\mathrm{C}(8)$ & $108.3(5)$ & $\mathrm{O}(4)-\mathrm{C}(17)-\mathrm{C}(16)$ & $111.0(5)$ \\
\hline $\mathrm{C}(9)-\mathrm{C}(8)-\mathrm{C}(13)$ & $120.0(6)$ & $\mathrm{C}(19)-\mathrm{C}(18)-\mathrm{C}(16)$ & $113.3(5)$ \\
\hline $\mathrm{C}(9)-\mathrm{C}(8)-\mathrm{C}(7)$ & $131.1(6)$ & $\mathrm{C}(19)-\mathrm{C}(18)-\mathrm{H}(18 \mathrm{~A})$ & 108.9 \\
\hline $\mathrm{C}(13)-\mathrm{C}(8)-\mathrm{C}(7)$ & $108.9(5)$ & $\mathrm{C}(16)-\mathrm{C}(18)-\mathrm{H}(18 \mathrm{~A})$ & 108.9 \\
\hline $\mathrm{C}(8)-\mathrm{C}(9)-\mathrm{C}(10)$ & 119.7(6) & $\mathrm{C}(19)-\mathrm{C}(18)-\mathrm{H}(18 \mathrm{~B})$ & 108.9 \\
\hline $\mathrm{C}(8)-\mathrm{C}(9)-\mathrm{H}(9)$ & 120.2 & $\mathrm{C}(16)-\mathrm{C}(18)-\mathrm{H}(18 \mathrm{~B})$ & 108.9 \\
\hline $\mathrm{C}(10)-\mathrm{C}(9)-\mathrm{H}(9)$ & 120.2 & $\mathrm{H}(18 \mathrm{~A})-\mathrm{C}(18)-\mathrm{H}(18 \mathrm{~B})$ & 107.7 \\
\hline $\mathrm{C}(9)-\mathrm{C}(10)-\mathrm{C}(11)$ & $120.9(6)$ & $\mathrm{C}(20)-\mathrm{C}(19)-\mathrm{C}(24)$ & $118.8(6)$ \\
\hline $\mathrm{C}(9)-\mathrm{C}(10)-\mathrm{H}(10)$ & 119.6 & $C(20)-C(19)-C(18)$ & $122.0(6)$ \\
\hline $\mathrm{C}(11)-\mathrm{C}(10)-\mathrm{H}(10)$ & 119.6 & $\mathrm{C}(24)-\mathrm{C}(19)-\mathrm{C}(18)$ & $119.2(6)$ \\
\hline $\mathrm{C}(10)-\mathrm{C}(11)-\mathrm{C}(12)$ & $120.0(6)$ & $\mathrm{C}(21)-\mathrm{C}(20)-\mathrm{C}(19)$ & $121.3(6)$ \\
\hline $\mathrm{C}(10)-\mathrm{C}(11)-\mathrm{H}(11)$ & 120.0 & $\mathrm{C}(21)-\mathrm{C}(20)-\mathrm{H}(20)$ & 119.3 \\
\hline $\mathrm{C}(12)-\mathrm{C}(11)-\mathrm{H}(11)$ & 120.0 & $\mathrm{C}(19)-\mathrm{C}(20)-\mathrm{H}(20)$ & 119.3 \\
\hline $\mathrm{C}(13)-\mathrm{C}(12)-\mathrm{C}(11)$ & $119.7(6)$ & $\mathrm{C}(20)-\mathrm{C}(21)-\mathrm{C}(22)$ & 119.2(6) \\
\hline $\mathrm{C}(13)-\mathrm{C}(12)-\mathrm{H}(12)$ & 120.1 & $\mathrm{C}(20)-\mathrm{C}(21)-\mathrm{H}(21)$ & 120.4 \\
\hline $\mathrm{C}(11)-\mathrm{C}(12)-\mathrm{H}(12)$ & 120.1 & $\mathrm{C}(22)-\mathrm{C}(21)-\mathrm{H}(21)$ & 120.4 \\
\hline $\mathrm{C}(12)-\mathrm{C}(13)-\mathrm{C}(8)$ & $119.8(6)$ & $\mathrm{C}(23)-\mathrm{C}(22)-\mathrm{C}(21)$ & $120.8(6)$ \\
\hline $\mathrm{C}(12)-\mathrm{C}(13)-\mathrm{C}(1)$ & $130.0(5)$ & $\mathrm{C}(23)-\mathrm{C}(22)-\mathrm{C}(25)$ & $120.6(7)$ \\
\hline $\mathrm{C}(8)-\mathrm{C}(13)-\mathrm{C}(1)$ & $110.1(5)$ & $\mathrm{C}(21)-\mathrm{C}(22)-\mathrm{C}(25)$ & $118.7(7)$ \\
\hline $\mathrm{O}(1)-\mathrm{C}(14)-\mathrm{C}(1)$ & $111.4(5)$ & $\mathrm{C}(22)-\mathrm{C}(23)-\mathrm{C}(24)$ & $119.5(6)$ \\
\hline $\mathrm{O}(1)-\mathrm{C}(14)-\mathrm{H}(14 \mathrm{~A})$ & 109.4 & $\mathrm{C}(22)-\mathrm{C}(23)-\mathrm{H}(23)$ & 120.2 \\
\hline $\mathrm{C}(1)-\mathrm{C}(14)-\mathrm{H}(14 \mathrm{~A})$ & 109.4 & $\mathrm{C}(24)-\mathrm{C}(23)-\mathrm{H}(23)$ & 120.2 \\
\hline
\end{tabular}


Table 4. Anisotropic displacement parameters $\left(\AA^{2} \times 10^{3}\right)$ for nilw106. The anisotropic displacement factor exponent takes the form: $-2 \pi^{2}\left[h^{2} a^{* 2} U_{11}+\ldots+2 h k a^{*} b^{*} U_{12}\right]$

\begin{tabular}{|c|c|c|c|c|c|c|}
\hline & $\mathrm{U}_{11}$ & $\mathrm{U}_{22}$ & $\mathrm{U}_{33}$ & $\mathrm{U}_{23}$ & $\mathrm{U}_{13}$ & $\mathrm{U}_{12}$ \\
\hline O1 & $20(2)$ & $36(2)$ & $53(3)$ & $1(2)$ & $3(2)$ & $1(2)$ \\
\hline $\mathrm{O} 2$ & $20(2)$ & $34(2)$ & $54(3)$ & $6(2)$ & $1(2)$ & $-1(2)$ \\
\hline $\mathrm{O} 3$ & $45(3)$ & $47(3)$ & $41(3)$ & $11(2)$ & $2(2)$ & $-3(2)$ \\
\hline $\mathrm{O} 4$ & $29(3)$ & $49(3)$ & $43(3)$ & $10(2)$ & $-3(2)$ & $5(2)$ \\
\hline N1 & $17(2)$ & $41(3)$ & $48(3)$ & $5(3)$ & $3(3)$ & $-7(2)$ \\
\hline $\mathrm{C} 1$ & $22(3)$ & $39(4)$ & $39(4)$ & 1(3) & $-3(3)$ & $-5(3)$ \\
\hline $\mathrm{C} 2$ & $26(4)$ & $36(4)$ & $35(4)$ & $-6(3)$ & $-8(3)$ & $-6(3)$ \\
\hline $\mathrm{C} 3$ & $37(4)$ & $43(4)$ & $47(4)$ & $1(4)$ & $-4(3)$ & $-5(3)$ \\
\hline $\mathrm{C} 4$ & $43(4)$ & $42(4)$ & $41(4)$ & $6(3)$ & $-4(3)$ & $-9(4)$ \\
\hline $\mathrm{C} 5$ & $44(4)$ & $60(5)$ & $36(4)$ & $-3(4)$ & 1(3) & $-13(4)$ \\
\hline C6 & $33(4)$ & $53(4)$ & $38(4)$ & $-6(4)$ & $1(3)$ & $-2(4)$ \\
\hline $\mathrm{C} 7$ & $26(4)$ & $46(4)$ & $33(4)$ & $-4(3)$ & $-1(3)$ & $-5(3)$ \\
\hline $\mathrm{C} 8$ & $24(4)$ & $47(4)$ & $40(4)$ & $-7(3)$ & $2(3)$ & $-5(3)$ \\
\hline C9 & $28(4)$ & $46(4)$ & $42(4)$ & $-6(3)$ & $3(3)$ & $5(4)$ \\
\hline $\mathrm{C} 10$ & $35(4)$ & $37(4)$ & $55(4)$ & $-4(4)$ & $-10(4)$ & $4(4)$ \\
\hline $\mathrm{C} 11$ & $36(4)$ & $37(4)$ & $46(4)$ & 2(3) & $-2(4)$ & $-3(3)$ \\
\hline $\mathrm{C} 12$ & $33(4)$ & $33(4)$ & $46(4)$ & $-2(3)$ & $2(3)$ & $0(3)$ \\
\hline $\mathrm{C} 13$ & $25(3)$ & $31(4)$ & $41(4)$ & $-2(3)$ & $-3(3)$ & $-2(3)$ \\
\hline $\mathrm{C} 14$ & $22(3)$ & $30(3)$ & $46(4)$ & $-2(3)$ & $2(3)$ & $3(3)$ \\
\hline $\mathrm{C} 15$ & $22(3)$ & $37(4)$ & $37(4)$ & $-4(3)$ & $-2(3)$ & $2(3)$ \\
\hline $\mathrm{C} 16$ & $20(3)$ & $34(3)$ & $57(4)$ & $2(3)$ & $-2(3)$ & $-1(3)$ \\
\hline $\mathrm{C} 17$ & $32(4)$ & $38(4)$ & $39(4)$ & $2(3)$ & $-1(3)$ & $-7(3)$ \\
\hline $\mathrm{C} 18$ & $32(4)$ & $43(4)$ & 41(4) & $7(3)$ & $-1(3)$ & $3(3)$ \\
\hline $\mathrm{C} 19$ & $27(3)$ & $40(4)$ & $45(4)$ & $3(3)$ & $2(3)$ & $6(3)$ \\
\hline $\mathrm{C} 20$ & $36(4)$ & $38(4)$ & $48(4)$ & $4(3)$ & $5(3)$ & $2(3)$ \\
\hline $\mathrm{C} 21$ & $45(5)$ & $36(4)$ & $47(4)$ & $4(3)$ & $7(4)$ & $3(4)$ \\
\hline $\mathrm{C} 22$ & $38(4)$ & $42(4)$ & $40(4)$ & $-11(3)$ & $16(3)$ & $-16(4)$ \\
\hline $\mathrm{C} 23$ & $37(4)$ & $53(5)$ & $40(4)$ & $-4(4)$ & 1(3) & $-4(4)$ \\
\hline $\mathrm{C} 24$ & $42(4)$ & 41(4) & $44(4)$ & $-5(3)$ & $2(3)$ & $10(4)$ \\
\hline $\mathrm{C} 25$ & $68(6)$ & $65(5)$ & $43(4)$ & $0(4)$ & $10(4)$ & $-12(6)$ \\
\hline N2 & $72(8)$ & $83(8)$ & $45(6)$ & $-7(6)$ & $7(6)$ & $-25(8)$ \\
\hline
\end{tabular}




\begin{tabular}{lcccccc} 
N3 & $68(6)$ & $65(5)$ & $43(4)$ & $0(4)$ & $10(4)$ & $-12(6)$ \\
O5 & $34(10)$ & $81(13)$ & $51(10)$ & $0(10)$ & $-4(8)$ & $-31(9)$ \\
O6 & $52(11)$ & $40(9)$ & $49(9)$ & $3(8)$ & $-20(9)$ & $-5(9)$ \\
\hline
\end{tabular}


Table 5. Hydrogen coordinates $\left(\times 10^{4}\right)$ and isotropic displacement parameters $\left(\AA^{2} \times 10^{3}\right)$ for nilw106.

\begin{tabular}{|c|c|c|c|c|}
\hline & $\mathrm{x}$ & $\mathrm{y}$ & z & $\mathrm{U}(\mathrm{eq})$ \\
\hline $\mathrm{H} 4 \mathrm{O}$ & -5695 & 2283 & 293 & 60 \\
\hline H1N & 724 & 1447 & 1147 & 42 \\
\hline H1 & 2033 & -1309 & 803 & 40 \\
\hline $\mathrm{H} 3$ & 3060 & 313 & 192 & 51 \\
\hline $\mathrm{H} 4$ & 5691 & 777 & -547 & 50 \\
\hline H5 & 9044 & 15 & -753 & 56 \\
\hline H6 & 9868 & -1229 & -243 & 50 \\
\hline H9 & 10008 & -2573 & 478 & 46 \\
\hline H10 & 9417 & -3655 & 1168 & 51 \\
\hline H11 & 6106 & -3677 & 1772 & 48 \\
\hline H12 & 3357 & -2588 & 1700 & 45 \\
\hline $\mathrm{H} 14 \mathrm{~A}$ & 2465 & -923 & 1812 & 39 \\
\hline H14B & 5063 & -590 & 1702 & 39 \\
\hline H16 & -3753 & 779 & 1187 & 44 \\
\hline H18A & -5273 & 1881 & 1790 & 47 \\
\hline H18B & -3291 & 1400 & 2160 & 47 \\
\hline $\mathrm{H} 20$ & -4560 & 3342 & 1450 & 49 \\
\hline $\mathrm{H} 21$ & -2453 & 4580 & 1550 & 51 \\
\hline $\mathrm{H} 23$ & 2415 & 3340 & 2520 & 52 \\
\hline $\mathrm{H} 24$ & 278 & 2092 & 2416 & 51 \\
\hline
\end{tabular}


Table 6. Torsion angles $\left[{ }^{\circ}\right]$ for nilw106.

\begin{tabular}{|c|c|c|c|}
\hline $\mathrm{C} 13-\mathrm{C} 1-\mathrm{C} 2-\mathrm{C} 3$ & $176.5(6)$ & $\mathrm{C} 2-\mathrm{C} 1-\mathrm{C} 13-\mathrm{C} 8$ & $4.1(6)$ \\
\hline $\mathrm{C} 14-\mathrm{C} 1-\mathrm{C} 2-\mathrm{C} 3$ & $-65.0(8)$ & $\mathrm{C} 14-\mathrm{C} 1-\mathrm{C} 13-\mathrm{C} 8$ & $-117.7(6)$ \\
\hline $\mathrm{C} 13-\mathrm{C} 1-\mathrm{C} 2-\mathrm{C} 7$ & $-3.4(6)$ & C15-O1-C14-C1 & $87.8(6)$ \\
\hline $\mathrm{C} 14-\mathrm{C} 1-\mathrm{C} 2-\mathrm{C} 7$ & $115.0(6)$ & C13-C1-C14-O1 & $167.2(4)$ \\
\hline $\mathrm{C} 7-\mathrm{C} 2-\mathrm{C} 3-\mathrm{C} 4$ & $-1.6(9)$ & $\mathrm{C} 2-\mathrm{C} 1-\mathrm{C} 14-\mathrm{O} 1$ & $52.6(7)$ \\
\hline $\mathrm{C} 1-\mathrm{C} 2-\mathrm{C} 3-\mathrm{C} 4$ & $178.4(6)$ & C16-N1-C15-O2 & $-1.2(10)$ \\
\hline $\mathrm{C} 2-\mathrm{C} 3-\mathrm{C} 4-\mathrm{C} 5$ & $1.1(9)$ & C16-N1-C15-O1 & $179.5(5)$ \\
\hline C3-C4-C5-C6 & $0.4(9)$ & C14-O1-C15-O2 & $7.2(9)$ \\
\hline $\mathrm{C} 4-\mathrm{C} 5-\mathrm{C} 6-\mathrm{C} 7$ & $-1.2(9)$ & C14-O1-C15-N1 & $-173.5(5)$ \\
\hline C5-C6-C7-C2 & $0.6(9)$ & C15-N1-C16-C17 & $-140.9(5)$ \\
\hline $\mathrm{C} 5-\mathrm{C} 6-\mathrm{C} 7-\mathrm{C} 8$ & $179.5(6)$ & C15-N1-C16-C18 & $96.5(6)$ \\
\hline $\mathrm{C} 3-\mathrm{C} 2-\mathrm{C} 7-\mathrm{C} 6$ & $0.8(9)$ & N1-C16-C17-O3 & $-8.0(9)$ \\
\hline $\mathrm{C} 1-\mathrm{C} 2-\mathrm{C} 7-\mathrm{C} 6$ & $-179.2(5)$ & $\mathrm{C} 18-\mathrm{C} 16-\mathrm{C} 17-\mathrm{O} 3$ & $115.0(7)$ \\
\hline $\mathrm{C} 3-\mathrm{C} 2-\mathrm{C} 7-\mathrm{C} 8$ & $-178.3(5)$ & N1-C16-C17-O4 & $174.1(5)$ \\
\hline $\mathrm{C} 1-\mathrm{C} 2-\mathrm{C} 7-\mathrm{C} 8$ & $1.7(6)$ & $\mathrm{C} 18-\mathrm{C} 16-\mathrm{C} 17-\mathrm{O} 4$ & $-62.9(6)$ \\
\hline C6-C7-C8-C9 & $0.9(11)$ & N1-C16-C18-C19 & $62.4(7)$ \\
\hline C2-C7-C8-C9 & $179.9(6)$ & C17-C16-C18-C19 & $-59.1(6)$ \\
\hline C6-C7-C8-C13 & $-178.0(6)$ & C16-C18-C19-C20 & $87.4(7)$ \\
\hline C2-C7-C8-C13 & $1.0(6)$ & C16-C18-C19-C24 & $-90.3(7)$ \\
\hline C13-C8-C9-C10 & $-0.6(9)$ & C24-C19-C20-C21 & $2.0(9)$ \\
\hline C7-C8-C9-C10 & $-179.4(6)$ & C18-C19-C20-C21 & $-175.7(6)$ \\
\hline C8-C9-C10-C11 & $0.8(9)$ & $\mathrm{C} 19-\mathrm{C} 20-\mathrm{C} 21-\mathrm{C} 22$ & $-0.4(10)$ \\
\hline C9-C10-C11-C12 & $-0.5(9)$ & $\mathrm{C} 20-\mathrm{C} 21-\mathrm{C} 22-\mathrm{C} 23$ & $-1.4(9)$ \\
\hline C10-C11-C12-C13 & $0.0(9)$ & $\mathrm{C} 20-\mathrm{C} 21-\mathrm{C} 22-\mathrm{C} 25$ & $178.0(6)$ \\
\hline C11-C12-C13-C8 & $0.2(9)$ & $\mathrm{C} 21-\mathrm{C} 22-\mathrm{C} 23-\mathrm{C} 24$ & $1.4(9)$ \\
\hline C11-C12-C13-C1 & $-176.9(6)$ & $\mathrm{C} 25-\mathrm{C} 22-\mathrm{C} 23-\mathrm{C} 24$ & $-178.0(6)$ \\
\hline C9-C8-C13-C12 & $0.1(9)$ & C22-C23-C24-C19 & $0.3(9)$ \\
\hline C7-C8-C13-C12 & 179.1(5) & C20-C19-C24-C23 & $-2.0(9)$ \\
\hline C9-C8-C13-C1 & $177.7(5)$ & C18-C19-C24-C23 & $175.8(6)$ \\
\hline $\mathrm{C} 7-\mathrm{C} 8-\mathrm{C} 13-\mathrm{C} 1$ & $-3.3(7)$ & $\mathrm{C} 23-\mathrm{C} 22-\mathrm{C} 25-\mathrm{N} 2$ & $132(13)$ \\
\hline $\mathrm{C} 2-\mathrm{C} 1-\mathrm{C} 13-\mathrm{C} 12$ & $-178.7(6)$ & $\mathrm{C} 21-\mathrm{C} 22-\mathrm{C} 25-\mathrm{N} 2$ & $-47(13)$ \\
\hline C14-C1-C13-C12 & $59.6(8)$ & & \\
\hline
\end{tabular}


Table 7. Hydrogen bonds and close contacts for nilw106 [ $\AA$ and $\left.{ }^{\circ}\right]$.

\begin{tabular}{lcccc}
\hline D-H...A & d(D-H) & $d(H \ldots . A)$ & $d(D \ldots A)$ & $<$ (DHA) \\
\hline O4-H4O...O3\#1 & 0.84 & 1.81 & $2.644(6)$ & 169.4 \\
N1-H1N...O4\#2 & 0.88 & 2.63 & $3.460(6)$ & 156.7 \\
\hline
\end{tabular}

Symmetry transformations used to generate equivalent atoms:

$\# 1 \mathrm{x}-1 / 2,-\mathrm{y}+1 / 2,-\mathrm{z} \quad \# 2 \mathrm{x}+1, \mathrm{y}, \mathrm{z}$ 